\title{
Free electron laser polarization control with interfering crossed polarized fields
}

\author{
Eugenio Ferrari, ${ }^{1, *}$ Eléonore Roussel, ${ }^{1, \dagger}$ Jens Buck, ${ }^{2, \ddagger}$ Carlo Callegari, ${ }^{1}$ Riccardo Cucini, ${ }^{1}$ \\ Giovanni De Ninno, ${ }_{1,3}$ Bruno Diviacco, ${ }^{1}$ David Gauthier, ${ }^{1,}$ Luca Giannessi, ${ }^{1,5}$ Leif Glaser, ${ }^{4}$ \\ Gregor Hartmann, ${ }^{4, \|}$ Giuseppe Penco, ${ }^{1}$ Frank Scholz, ${ }^{4}$ Joern Seltmann, ${ }^{4}$ Ivan Shevchuk,${ }^{4}$ \\ Jens Viefhaus, ${ }^{4, \mathbb{T}}$ Marco Zangrando, ${ }^{1,6}$ and Enrico Massimiliano Allaria ${ }^{1}$ \\ ${ }^{1}$ Elettra-Sincrotrone Trieste S.C.p.A., Strada Statale 14-km 163,5 in AREA Science Park, \\ 34149 Basovizza, Trieste, Italy \\ ${ }^{2}$ European XFEL GmbH, Holzkoppel 4, 22869 Schenefeld, Germany \\ ${ }^{3}$ Laboratory of Quantum Optics, University of Nova Gorica, 5000 Nova Gorica, Slovenia \\ ${ }^{4}$ Deutsches Elektronen-Synchrotron DESY, FS-PE, Notkestrasse 85, 22607 Hamburg, Germany \\ ${ }^{5}$ ENEA, via Enrico Fermi 45, 00044 Frascati, Roma, Italy \\ ${ }^{6}$ IOM-CNR, Strada Statale 14-km 163, 5 in AREA Science Park, 34149 Basovizza, Trieste, Italy
}

(Received 9 January 2019; published 23 August 2019)

\begin{abstract}
Free electron lasers emit powerful and coherent radiation in a wide wavelength range extending to hard $\mathrm{x}$-rays. This radiation is also characterized by a high degree of polarization that is generally linear and depends on the undulator properties. The possibility of controlling the polarization state of the radiation is an important option for free electron lasers that is critical for a large class of experiments. Such control can be achieved using variable polarization undulators or alternatively via the crossed polarized undulator scheme. We report the results of an extensive study for the characterization of the crossed-polarized undulator scheme in a number of different configurations. A simple model, based on Gaussian mode beam propagation, is presented and used to reproduce the experimental results obtained at the seeded free electron laser FERMI. A good agreement is found between the model and the experiment allowing us to understand the impact of the wavefront properties of the radiation coming from the consecutive undulators on the output radiation. The model is used not only for characterizing the control of the polarization but also for the control of the transverse mode.
\end{abstract}

DOI: 10.1103/PhysRevAccelBeams.22.080701

\section{INTRODUCTION}

While variable polarization control is widespread for synchrotron-based light sources [1-6], it is still limited for present day free electron lasers (FELs). This is mainly due

*Present address: Paul Scherrer Institut, CH-5232 Villigen PSI, Switzerland.

†Present address: Université de Lille, CNRS, UMR 8523PhLAM-Physique des Lasers, Atomes et Molécules, F-59000, Lille, France.

${ }^{\ddagger}$ Present address: Deutsches Elektronen-Synchrotron DESY, FS-PE, Notkestrasse 85, D-22607 Hamburg, Germany.

${ }^{\S}$ Present address: Atomic Energy and Alternative Energies Commission (CEA), Centre d'Etudes de Saclay, F-91191 Gifsur-Yvette, France.

"Present address: Institut für Physik und CINSaT, Universität Kassel, Heinrich-Plett-Strasse 40, D-34132 Kassel, Germany.

Present address: Helmholtz-Zentrum Berlin für Materialien und Energie GmbH, Albert-Einstein-Straße 15, D-12489 Berlin, Germany.

Published by the American Physical Society under the terms of the Creative Commons Attribution 4.0 International license. Further distribution of this work must maintain attribution to the author(s) and the published article's title, journal citation, and DOI. to technological and cost reasons related to the development of suitable undulators capable of sustaining the FEL process. Alternative solutions for achieving polarization control, in particular for SASE FELs in the X-ray wavelength range employing linearly-polarized undulators, have been proposed and studied in the last decades [7-10]. One of the most attractive possibilities relies on the coherent superposition of radiation generated by orthogonally polarized undulators [8] in the so-called "crossed polarized undulators" (XPU) scheme.

The first demonstration of the XPU scheme was implemented on a synchrotron source [11] where the light from two linearly polarized undulators produced circularly polarized light which was then utilised to perform magnetic dichroism experiments [12]. The limited degree of polarization, $\sim 0.4$ reported in [11], was a priori determined by the partial coherence of the source and the wide bandwidth of 
the emission. In fact, the ideal source for the XPU setup must have a high degree of coherence and narrow bandwidth in order to enable significant constructive interference of the two electromagnetic waves emitted by the orthogonal undulators [8]. FEL sources represent an ideal candidate for the scheme because of their high transverse coherence; hence a high degree of polarization is expected [9].

When insertion devices with variable polarization are available $[13,14]$, the XPU scheme can be used to generate radiation with arbitrary polarization, or linearly polarized emission free of on-axis higher harmonic content. A schematic depiction of the XPU principle for different cross-polarization configuration is shown in Fig. 1. The XPU scheme offers the interesting possibility of extremely fast polarization switching by tuning of the electromagnetic phase shifters installed between the undulators. This capability is attractive for novel user experiments, as it does not require a change in the undulator tuning, which can be both time consuming and require additional electron beam optics optimization.

The XPU scheme has been successfully demonstrated on multiple FEL devices including oscillators operating in an optical klystron configuration [16] and amplifiers in both the low-gain regime at visible wavelengths [17] and the high-gain regime at XUV wavelengths [18]. The latter case was studied at the FEL-1 of FERMI and despite the high degree of coherence [19-21], the measured maximum degree of polarization was limited to $\sim 0.8$. This reduced degree of polarization with XPU from a nearly fully

(a)

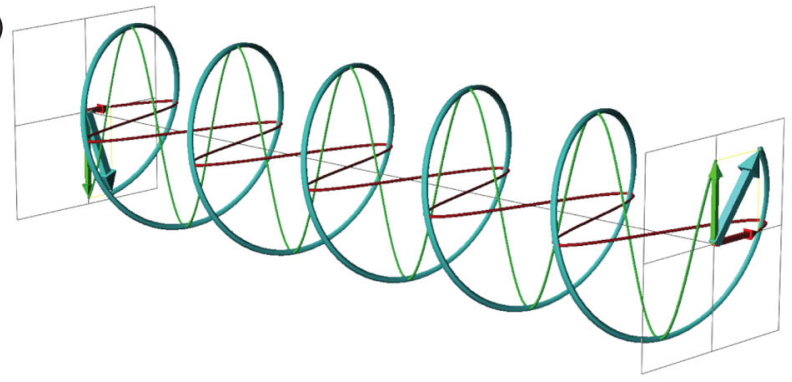

(b)

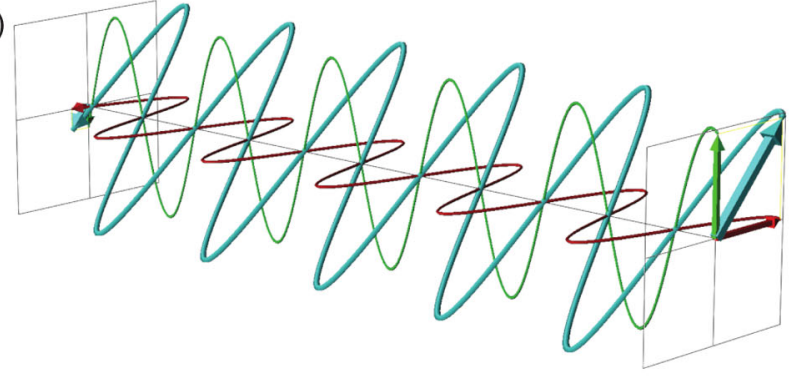

coherent source as FERMI, was attributed to the interference between the radiation produced by multiple undulators that are separated by a finite distance. For a better understanding of the XPU process, we have developed a suitable model that, by considering the free space diffraction of the radiation, reproduces the measured results. In this work additional XPU measurements are reported and compared with model predictions. The model is introduced in Sec. II together with a description of the used FERMI FEL-1 setup and it is used for reproducing the transverse pattern interference of coherent fields with same polarization produced by consecutive FEL undulators. A thorough experimental characterisation of the XPU scheme at FERMI is then presented in Sec. III and results are compared with model predictions. We conclude in Sec. IV with a short summary of our findings.

\section{EXPERIMENTAL SETUP AND MODEL}

For the experiments reported here, we operated the FERMI FEL-1 [22] at a wavelength of $23.8 \mathrm{~nm}$, corresponding to the 11th harmonic of the $261.1 \mathrm{~nm}$ external seed laser. The electron beam had an energy $\sim 1.45 \mathrm{GeV}$ and a $700 \mathrm{pC}$ charge compressed to a peak current of $650 \mathrm{~A}$. Additional parameters are reported in Table I; other e-beam and FEL parameters correspond to the standard values for FERMI and can be obtained from [23].

For the polarization measurements, data have been acquired using the e-TOF polarimeter [24] that is capable

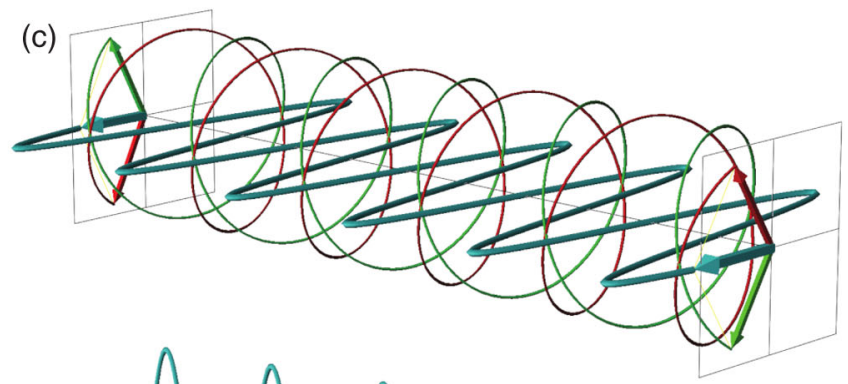

(d)

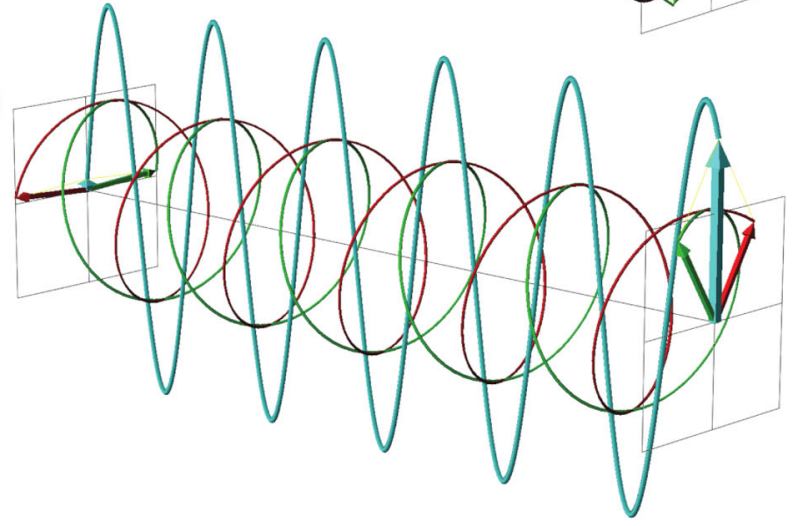

FIG. 1. Schematic depiction of the XPU scheme. (a) and (b) describe the superposition of two linearly polarized sources, LH (red line) and LV (green line) to obtain either circularly polarized, or linear polarization at an arbitrary direction (light blue line). (c) and (d) schematically illustrate the superposition of CR and CL sources (red and green curves, respectively) to produce linearly polarized light with arbitrary direction (light blue curve, along the vertical and horizontal direction in the image). Images obtained using [15]. 
TABLE I. Relevant parameters for the FEL configuration used in the experiment.

\begin{tabular}{lc}
\hline \hline Undulator length & $6 \times 2.4 \mathrm{~m}$ \\
Drift length & $1.3 \mathrm{~m}$ \\
FEL wavelength & $23.8 \mathrm{~nm}$ \\
Beam waist (RMS) & $100 \mu \mathrm{m}$ \\
FEL Rayleigh range & $1.32 \mathrm{~m}$ \\
\hline \hline
\end{tabular}

of characterizing the polarization of the radiation on a shotto-shot basis $[13,14]$. The polarimeter was installed at the back end of FERMI's LDM beamline [25], approximately $50 \mathrm{~cm}$ downstream of the nominal LDM endstation focal position. The diverging photon beam size at the measurement location was $>100 \mu \mathrm{m}$ in order to prevent higher order effects on the photoionization spectra. For details about the transmission at different wavelengths and for different polarization states, see $[13,14]$. At $23.8 \mathrm{~nm}$ wavelength, the beamline simulated transmission has a $4 \%$ difference between LH and LV polarization. The stability and reproducibility of the XPU scheme constitute one of the main concerns for its applicability to experiments [26]. Consequently we performed a careful characterisation of the statistical fluctuations of the FEL polarization properties.

\section{A. FERMI FEL-1}

The radiator for FEL-1 consists of six separate undulators that can be independently tuned in wavelength and polarization, allowing various possibilities to implement advanced schemes such as two pulse-two color $[19,27]$ or the crossed polarized undulator scheme [18]. The undulators are each 2.4-m long and separated by drift spaces of $\sim 1.3 \mathrm{~m}$ where quadrupole focusing, phase shifters, and diagnostics are located.

With the variable polarization APPLE-II undulators [28] used at FERMI, two separate groups of orthogonal linearly polarized undulators can be superposed to produce circular polarization, or vice versa. Moreover, the flexibility of the undulator setup allows one to use two different XPU configurations as illustrated in Figs. 2(b),(c). Similar to the original proposal [8], one can implement the crossed polarized undulator scheme by setting a first contiguous group of undulators to a given polarization state, and a second set of undulators to the corresponding orthogonal polarization [Fig. 2(b)]. A critical requirement for this configuration is that the intensity of the two polarized fields should be balanced as exactly as possible. Due to the increasing gain as the electrons travel along the radiator, this leads to an unequal number of undulators for the two sets. Another possibility [Fig. 2(c)] is the distributed XPU scheme $[29,30]$ in which the undulators are tuned with alternating polarization. In this case both orthogonal fields grow along the radiator chain allowing easier balance of the two field intensities. The distributed XPU scheme was also implemented on SPring-8 storage ring [31].

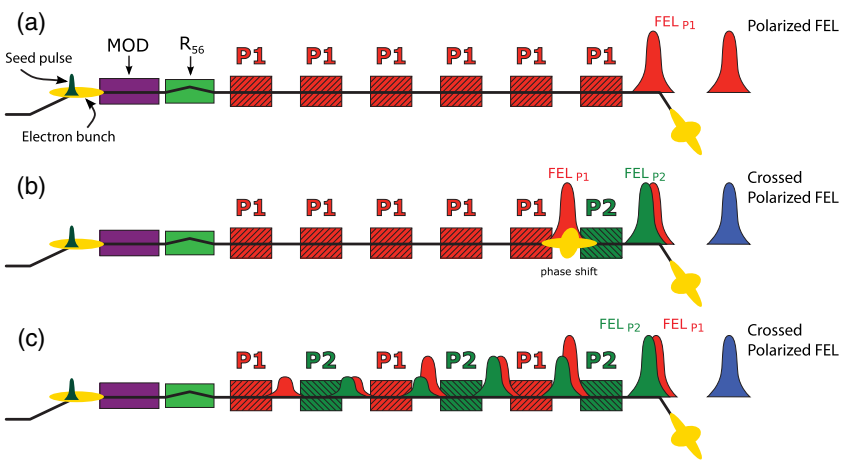

FIG. 2. Schematic representation of the FEL-1 setup. In the normal configuration (a) all the modules constituting the radiator are tuned to the same polarization state to emit polarized FEL radiation [circular right (CR), circular left (CL), linear vertical (LV) or linear horizontal (LH)]. The crossed polarized undulator scheme instead can be implemented as a superposition of two consecutive sources (b) or in a distributed scheme (c). Red and green represent undulators with two orthogonal polarizations (P1 and P2) that can be either $\mathrm{CR}-\mathrm{CL}$, or $\mathrm{LH}-\mathrm{LV}$ that produce FEL pulses with corresponding polarization properties $\left(\mathrm{FEL}_{\mathrm{P} 1}\right.$ and $\mathrm{FEL}_{\mathrm{P} 2}$ ). The labels MOD indicates the modulator undulator, $\mathrm{R}_{56}$ the dispersive section. Image adapted from [18].

The phase shifters installed in the drifts between undulators are critical systems. During normal FEL operations, careful phase shifter tuning is necessary for optimizing the FEL gain by maximizing the constructive interference of emission from consecutive undulators. For the XPU scheme, the setting of phase shifters consequently determines the final polarization state of the output FEL pulse.

\section{B. Theoretical model}

The XPU scheme relies on the generation of two orthogonal fields that must have similar characteristics despite being emitted from different sets of undulators. This requirement can be achieved when there is limited exponential growth of the bunching within a single undulator length. For a very high gain SASE FEL that starts from noise, a possible scheme is to place two orthogonally polarized undulators at the very end of the main undulator line [9]. For an externally seeded FEL such as FERMI where the initial microbunching level is much higher, coherent, and controllable, there is significantly more flexibility in optimizing the XPU scheme.

In both cases the FEL radiation is produced by a prebunched beam in relatively short undulators and is well described by a TEM $_{00}$ Gaussian mode, whose beam waist is determined by the electron beam size within the undulator and whose source point coincides approximately with the undulator center. In this approximation the radiation from different undulators can be described by Gaussian modes, each with different source points. As a result, given the different curvature of the two orthogonally polarized fields' wavefront at the target, the relative eikonal phase varies as one moves off axis (see Fig. 3). 


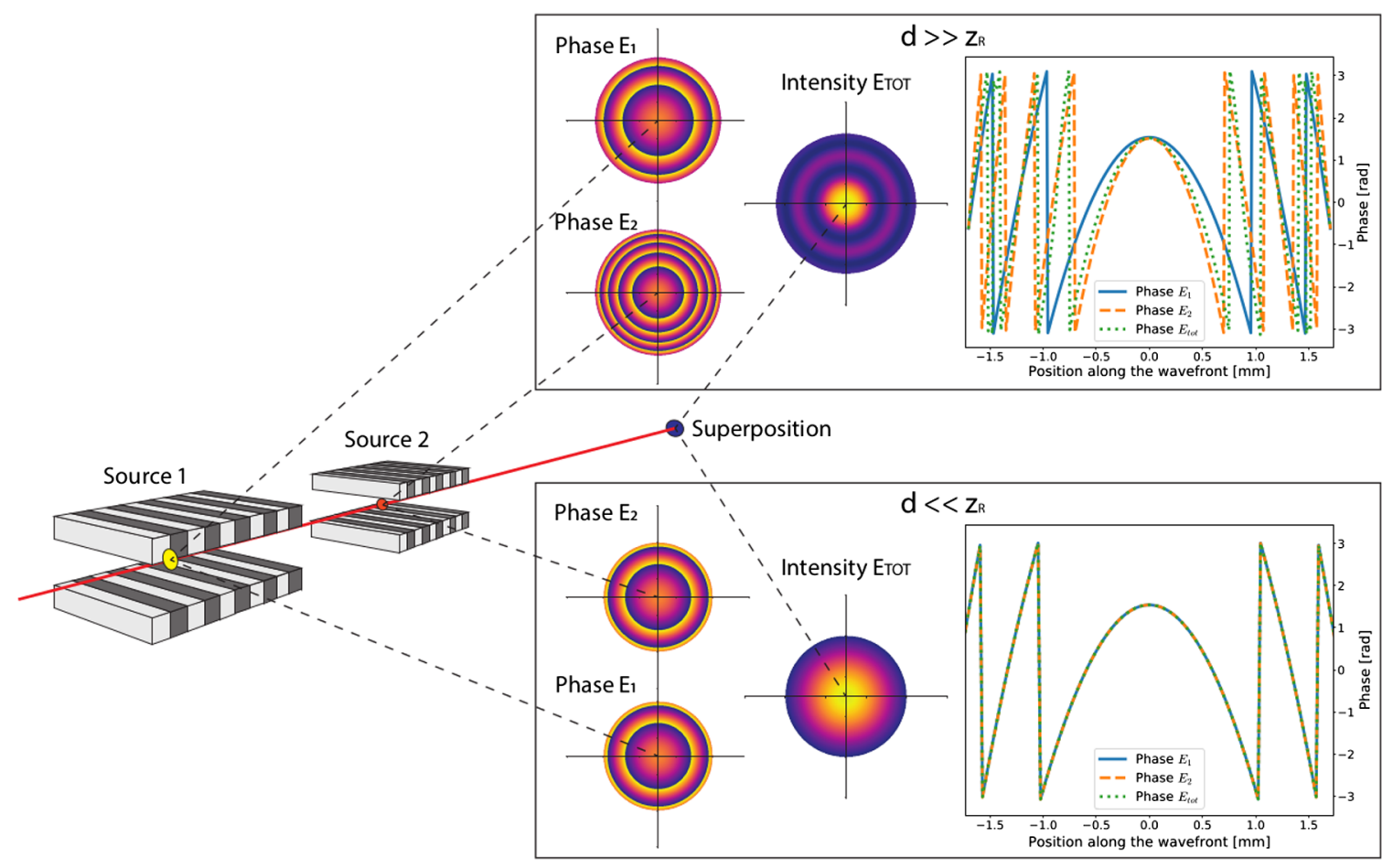

FIG. 3. Phases of the superimposed electric fields $\left(E_{1}\right.$ and $\left.E_{2}\right)$, together with the magnitude of the total electric field ( $\left.E_{\text {tot }}\right)$. Top: when the distance $d$ between the sources is larger than the Rayleigh length $z_{R}$ of the radiation, the two sources have different phase profiles at the interaction point, hence the superposition field exhibits phase differences along the wavefront. Bottom: when the distance between the sources is smaller than $z_{R}$ no significant phase shift is present transversely.

The fact that the phase difference between the two fields is not constant but changes off axis has an impact on the optimum setting of the phase shifter. In fact, for a value of the phase shifter that produces constructive interference for the two on-axis fields, there will be a locus off axis where the two fields will be in antiphase, and will interfere destructively; here the total field will show a relative minimum. The larger the distance $d$ between the two source points compared to the Rayleigh length of the radiation $z_{R}$, the larger the effect.

Evidence of this effect at FERMI can be observed in Fig. 4 where the transverse mode of FEL-1 operated at $23.8 \mathrm{~nm}$ is shown for three different values of the phase shifter between the last two undulators, both emitting at the same wavelength and polarization. We now outline a simple model whose predictions will then be compared to these experimental results.

\section{Model description}

For our model we consider the case where the bunching does not change significantly over the distance of a single undulator. This condition is not suitable for characterizing the exponential growth of the power in a SASE FEL where the gain guiding strongly affects the FEL mode evolution [32]. However it is very well suited for the FEL conditions used for the FERMI XPU experiments described before.
We treat the radiation emitted from each undulator as an independent Gaussian mode beam. The mode's waist location is placed at the center of the undulator segment, the waist size ( $w_{0}^{j}$ in the following) is assumed to be equal to the ( $z$-averaged) transverse electron beam dimension in the undulator. It can be estimated either knowing the Twiss parameters along the undulator chain, or by measuring the electron beam transverse size at each undulator exit as for the results reported in Sec. III. Finally, the intensity of each Gaussian mode can be estimated by measuring the contribution of each undulator to the overall FEL power via an FEL gain curve.

Adopting a Cartesian coordinate system $(x, y, z)$ with $z$ parallel to the propagation direction, the eikonal electric field of the $j$ th source can be decomposed into its orthogonal horizontal $(H)$ and vertical $(V)$ components:

$$
\begin{aligned}
E_{H}^{j}(x, y, z)= & E_{0, H}^{j} \frac{w_{0}^{j}}{w^{j}(z)} \exp \left[-\left(\frac{r}{w^{j}(z)}\right)^{2}\right. \\
& \left.-i k z-i k \frac{r^{2}}{2 R^{j}}+i \chi\right] \\
E_{V}^{j}(x, y, z)= & E_{0, V}^{j} \frac{w_{0}^{j}}{w^{j}(z)} \exp \left[-\left(\frac{r}{w^{j}(z)}\right)^{2}-i k z\right. \\
& \left.-i k \frac{r^{2}}{2 R^{j}}+i \chi+i \varphi_{H \rightarrow V}^{j}\right],
\end{aligned}
$$



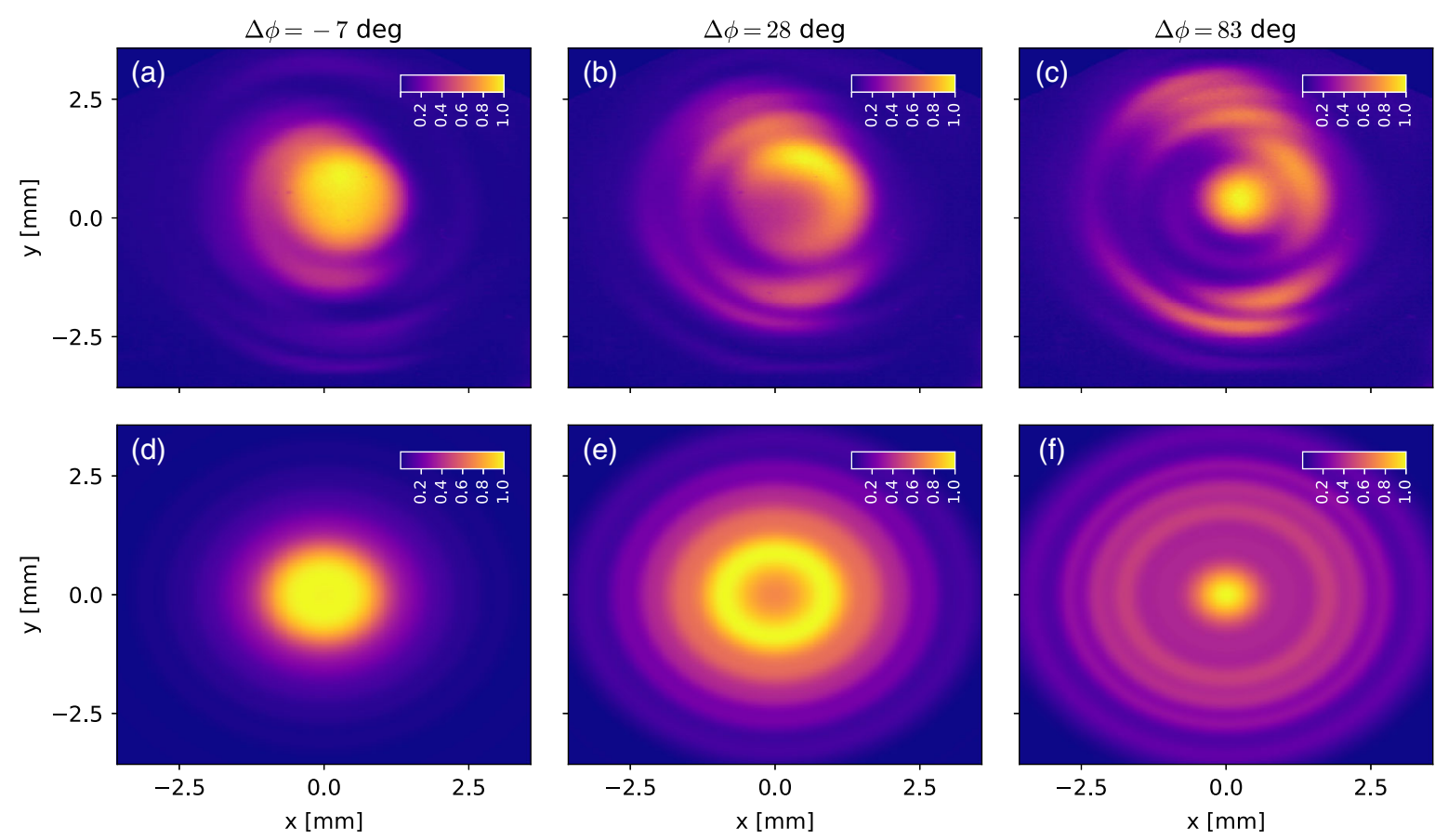

FIG. 4. Experimental FEL transverse spot profile for three different settings of the last phase shifter $\Delta_{\phi_{6}}$. (a) the phasing enhances the emission of on-axis Gaussian mode, (b) intermediate and (c) the net emission is mainly off-axis. (d, e, f) Model predicted transverse radiation profile. The transverse spot profile was measured at a distance of $\sim 60 \mathrm{~m}$ from the exit of the last undulator. For the simulations, the parameters were $\lambda=23.8 \mathrm{~nm}, w_{0}=100 \mu \mathrm{m}, d=3.7 \mathrm{~m}$, gain length $2.2 \mathrm{~m}$.

where

$$
z_{R}^{j}=\pi \frac{\left(w_{0}^{j}\right)^{2}}{\lambda}
$$

is the Rayleigh length,

$$
w^{j}(z)=w_{0}^{j} \sqrt{1+\left(\frac{z}{z_{R}^{j}}\right)^{2}}
$$

is the mode size at distance $z$ from the waist, $w_{0}^{j}$ the waist size, $k$ the wave number,

$$
R^{j}=z\left[1+\left(\frac{z_{R}^{j}}{z}\right)^{2}\right]
$$

is the radius of curvature, $r=\sqrt{x^{2}+y^{2}}$ the distance from the beam axis,

$$
\chi=\tan ^{-1}\left(\frac{z}{z_{R}^{j}}\right)
$$

is the Gouy phase [33] and finally $\varphi_{H \rightarrow V}^{j}$ is the relative phase between the horizontal and vertical components of the $j$ th source, defining its polarization. Equation (1) implicitly presumes that all sources are strictly coaxial and have no tilt.

As an example, a circularly polarized source can be written as $E_{0, H}^{j}=E_{0, V}^{j}$ and $\varphi_{H \rightarrow V}^{j}=90 \mathrm{deg}(\mathrm{CR})$ or
$\varphi_{H \rightarrow V}^{j}=-90 \mathrm{deg}$ (CL), while a linear horizontal (LH) polarized source can be written as $E_{0, H}^{j}=$ constant, $E_{0, V}^{j}=0$, $\varphi_{H \rightarrow V}^{j}=0$ deg.

The horizontal and vertical components of the resulting electric field can be written, according to the superposition principle, as

$$
E_{H, V}^{\mathrm{tot}}(x, y, z)=\sum_{j} E_{H, V}^{j}\left(x, y, z_{j}\right) \times \exp \left(i \Delta \phi_{j}\right),
$$

where $z_{j}$ is the distance of each source, i.e., each undulator center, from the measurement location. For convenience, we separate the phase contribution to each source in two parts. The first, $\varphi_{j}$, is the phase between the horizontal and vertical electric field components. It allows us to define a generic polarization state for each source, e.g., circular polarization. The second, $\Delta \phi_{j}$, is an additional phase shift between the different sources along the undulator chain. It can be varied experimentally by tuning the phase shifters between each undulator module.

For simplicity, here we ignore possible transverse misalignments of the sources relative to one another, although they can be included in the model. We also ignore any differential phase or intensity effects due to transport and focusing optics which we believe are small.

Equation (6) can be used for calculating the properties of the resulting electric field such as the Stokes parameters [34] at the measurement position. 
The dependence of the (normalized) Stokes parameters on the transverse position at a given $z$ location can be estimated by means of:

$S_{0}(x, y)=\left|E_{H}(x, y)\right|^{2}+\left|E_{V}(x, y)\right|^{2}=I_{\left(0^{\circ}\right)}(x, y)+I_{\left(90^{\circ}\right)}(x, y)$,

$S_{1}(x, y)=\left|E_{H}(x, y)\right|^{2}-\left|E_{V}(x, y)\right|^{2}=I_{\left(0^{\circ}\right)}(x, y)-I_{\left(90^{\circ}\right)}(x, y)$,

$S_{2}(x, y)=\Re\left(E_{H}(x, y) E_{V}(x, y)\right)=I_{\left(45^{\circ}\right)}(x, y)-I_{\left(135^{\circ}\right)}(x, y)$,

$S_{3}(x, y)=\Im\left(E_{H}(x, y) E_{V}(x, y)\right)=I_{R H}(x, y)-I_{L H}(x, y)$.

In Eq. (7) $I_{(\ldots)}(x, y)$ is the intensity for linearly polarized components over the indicated direction while $I_{R H}(x, y)$ and $I_{L H}(x, y)$ are the intensities of the circularly polarized components (right and left) of the fields.

The defined local Stokes parameters [Eq. (7)] determine the local polarized fraction of the light, $P(x, y)$ as

$$
P(x, y)=\frac{\sqrt{S_{1}(x, y)^{2}+S_{2}(x, y)^{2}+S_{3}(x, y)^{2}}}{S_{0}(x, y)}
$$

which represents the fractional intensity of the polarized component at each $x, y$ position along the grid on which we compute our model. We also define the local linear polarized fraction of the light $P_{l i n}(x, y)$ and the local direction of the linear polarization vector $\psi(x, y)$ as:

$$
\begin{aligned}
P_{\text {lin }}(x, y) & =\frac{\sqrt{S_{1}(x, y)^{2}+S_{2}(x, y)^{2}}}{S_{0}(x, y)}, \\
\psi(x, y) & =\frac{1}{2} \tan ^{-1}\left[\frac{S_{2}(x, y)}{S_{1}(x, y)}\right] .
\end{aligned}
$$

Finally, taking the integral of the local Stokes parameters over all the $(x, y)$ coordinates in the transverse plane, we can define the global Stokes parameters as:

$$
\begin{aligned}
& \boldsymbol{S}_{\mathbf{0}}=\left\langle S_{0}(x, y)\right\rangle, \\
& \boldsymbol{S}_{\mathbf{1}}=\left\langle S_{1}(x, y)\right\rangle, \\
& \boldsymbol{S}_{\mathbf{2}}=\left\langle S_{2}(x, y)\right\rangle, \\
& \boldsymbol{S}_{\mathbf{3}}=\left\langle S_{3}(x, y)\right\rangle,
\end{aligned}
$$

where $\langle\ldots\rangle$ indicates the integral over the transverse plane. From the total Stokes parameters we can again obtain the total polarization fraction $\boldsymbol{P}$, linear polarization fraction $\boldsymbol{P}_{\text {lin }}$ and direction of the linear polarization vector $\boldsymbol{\psi}$ in a similar way as done in Eqs. (8) and (9).

Let us now consider the simplest XPU configuration, namely just two collinear sources with orthogonal polarization states, LH and LV, with distinct source locations. In Figs. 5 and 6(a)-6(c) we report the distribution for the final pulse properties, i.e., intensity $I(x, y)$, degree of linear
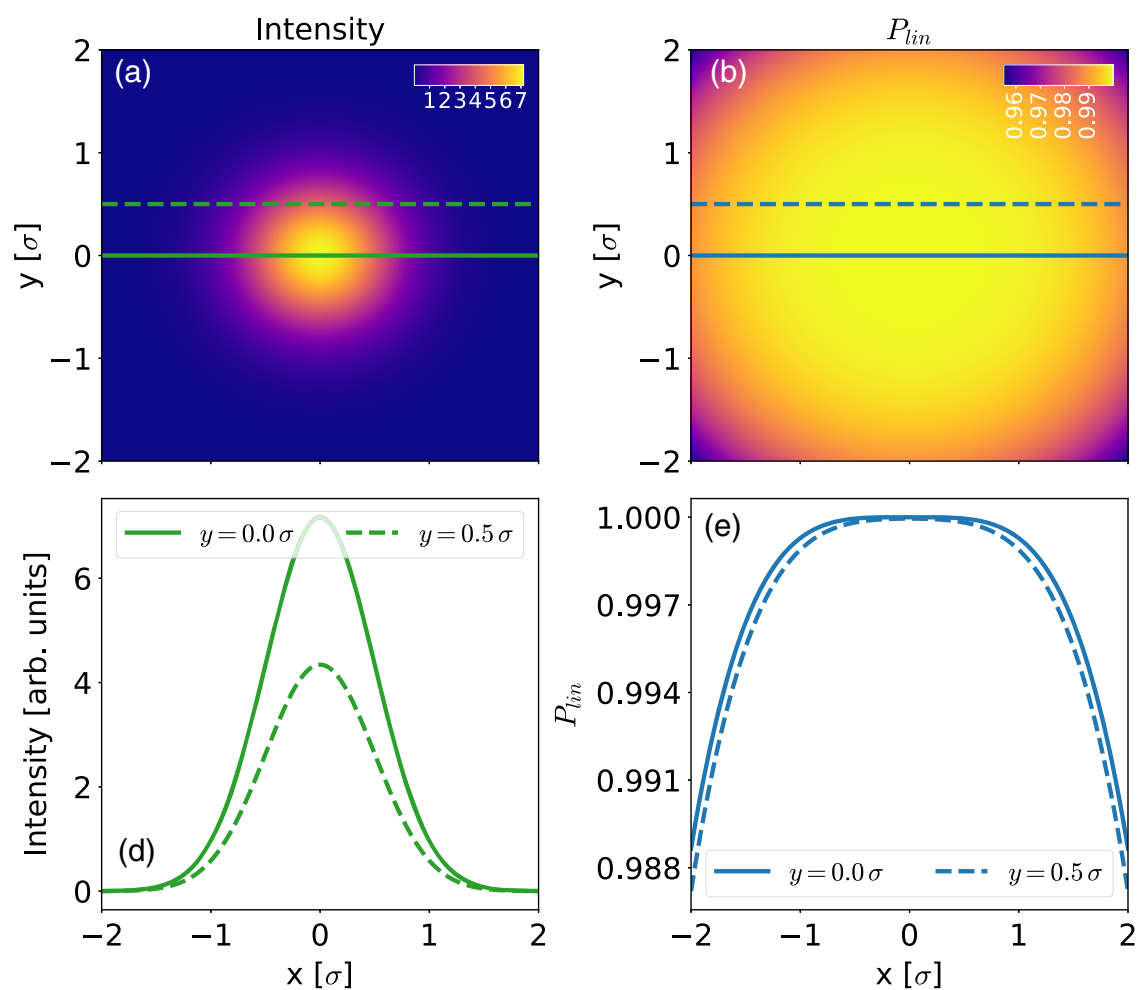
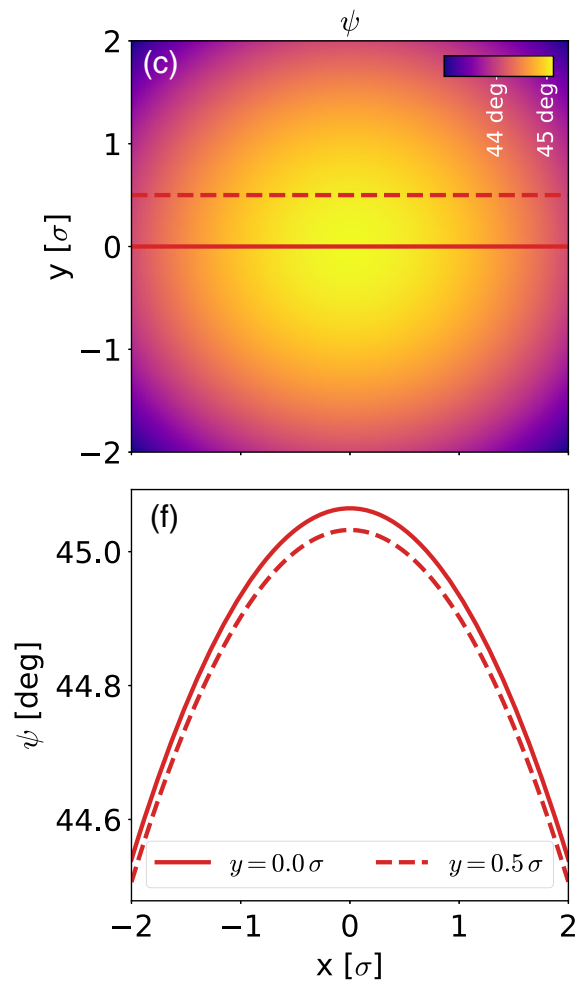

FIG. 5. False color representation of the far field intensity (a), degree (b) and direction (c) of linear polarization, for the superposition of two Gaussian beams with orthogonal polarization, namely linear horizontal and linear vertical, with phase shift an integer of the wavelength. The distance between the two sources is $0.03 z_{R}$. The above quantities are shown along the solid and dashed lines on the surfaces on the plots. The polarization properties of the radiation are in this case essentially constant along the wavefront of the radiation. 

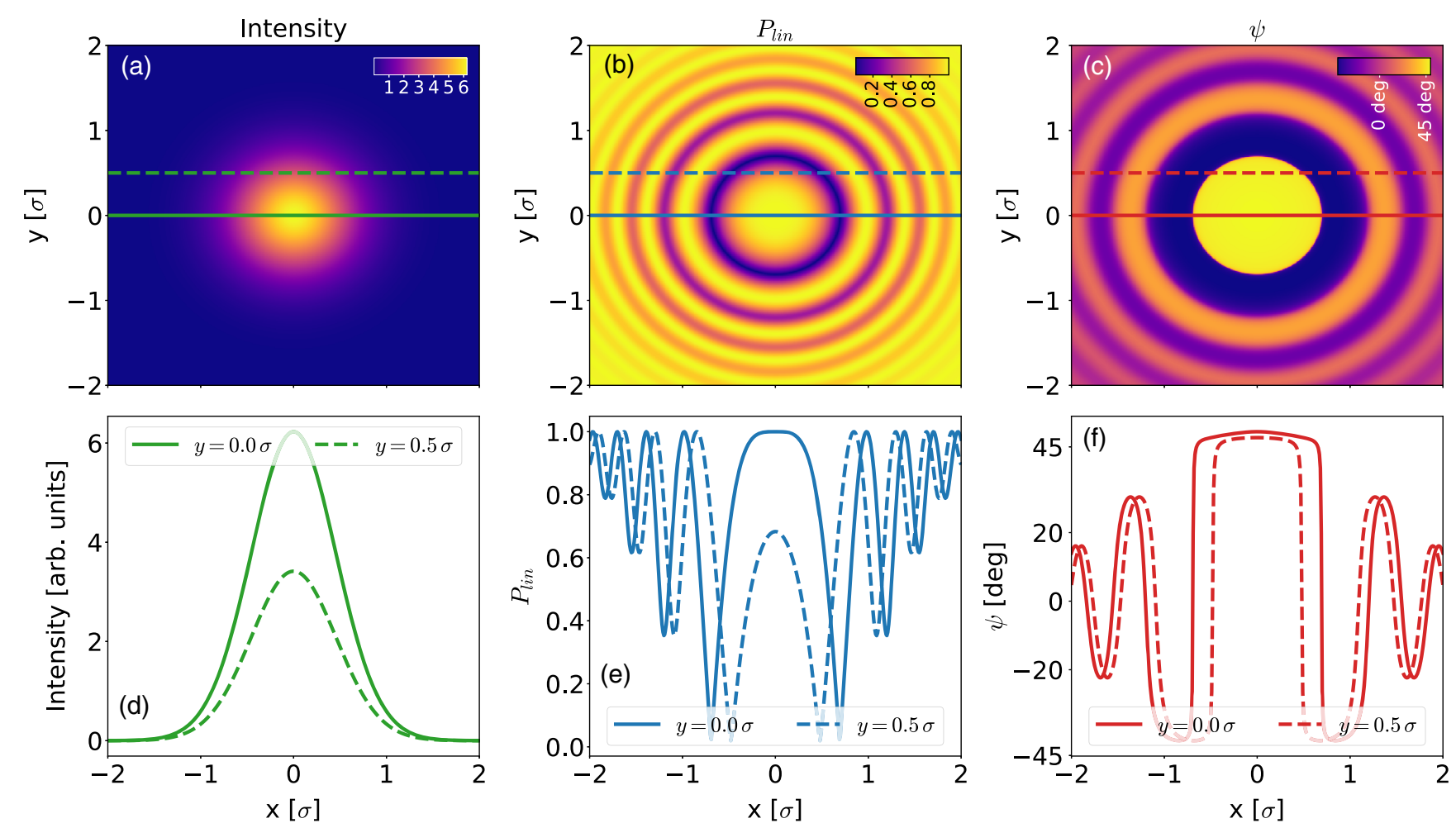

FIG. 6. False color representation of the far field intensity (a), degree (b) and direction (c) of linear polarization, for the superposition of two Gaussian beams with orthogonal polarization, namely linear horizontal and linear vertical, with phase shift an integer of the wavelength. The distance between the two sources is $3.0 z_{R}$. The above quantities are shown along the solid and dashed lines on the surfaces on the plots. One can see that the polarization properties are changing along the wavefront of the total field if the sources' separation is larger that the Rayleigh range of the radiation.

polarization $P_{l i n}(x, y)$, and polarization direction $\psi(x, y)$ calculated, using our model, over the transverse plane $(x, y)$ for two different source properties, respectively. The coordinates are scaled as function of the radiation size $\sigma$. Figures 5 and 6(d)-6(f) also show the equivalent curves representing the same quantities projected over $x$ at two specific $y$ positions within the pulse profiles.

Two different separation distances between the sources, which otherwise have the same intensity and size at waist are considered. These separations have been chosen to either be much smaller $\left(0.03 z_{R}\right)$, see Fig. 5, or much larger $\left(3.0 z_{R}\right)$, see Fig. 6, than the Rayleigh length of the radiation.

The interference of the two diffraction fields which is the origin of the difference between the two reported cases (Figs. 5 and 6) also has an impact on the maximum degree of total polarization $\boldsymbol{P}$ that can be obtained with the XPU scheme for two fully coherent pulses. We used the model to calculate the degree of total polarization for the whole pulse as a function of the distance between the two sources $d$, while keeping constant the other field parameters.

The results are reported in Fig. 7, where the distance has been scaled to the Rayleigh range of the radiation. One can clearly see that a high degree of polarization $\boldsymbol{P}$ is obtained when $d / z_{R}$ is (much) less than one, i.e., when the distance

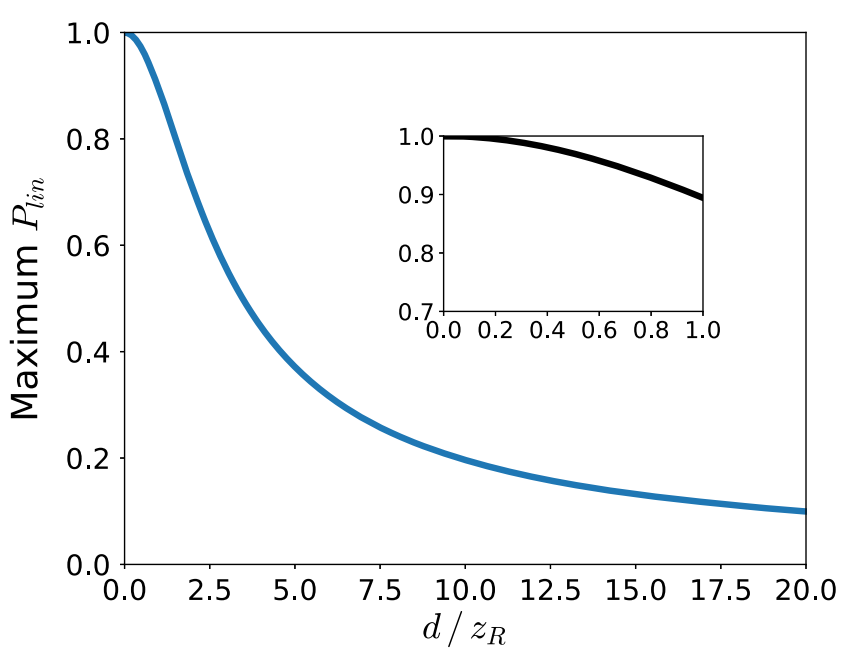

FIG. 7. Degree of linear polarization as a function of the distance between two cross-polarized sources, normalized by the Rayleigh range of the radiation. The two sources have the same amplitude and are completely linearly polarized. Insert: zoom for $d / z_{R}<1$. One can see that the maximum $\boldsymbol{P}_{\text {lin }}$ obtainable depends critically on the distance between the sources, as the phase difference along the wavefront becomes more critical if the distance becomes larger than the Rayleigh range. 
between the two sources is smaller than the Rayleigh range of the radiation. In this case the sources are interfering almost as two plane waves and the propagation effects can be neglected. When instead the separation distance is larger than $z_{R}$, the polarization properties of the radiation $\left[S_{i}(x, y)\right]$ drastically change radially over the transverse plane of the beam, leading to an overall decrease of the total degree of linear polarization $\boldsymbol{P}_{\text {lin }}$ for the whole beam. The radial dependence of the polarization properties is due to the variation of the relative phase and amplitude ratio between the two fields at the measurement location along the wavefront. By considering the average polarization of the overall beam spot, a decrease of $\boldsymbol{P}_{\text {lin }}$ is observed.

In the case of FERMI the distance between the centers of two successive undulators is few times larger than $z_{R}$, depending on the wavelength of the radiation and the electron beam properties along the undulator chain, see Table I for the parameters of the experiments discussed in the following. In the following we will focus on the case $d / z_{R}>1$, pertinent to FERMI. For convenience, the phase is reported in units of $\lambda$, i.e., a shift of $\lambda / 2$ corresponds to a phase shift of $180 \mathrm{deg}$.

\section{XPU with LV and LH fields}

When two linearly cross-polarized fields are superposed, the resulting radiation has a polarization state that depends on the phase between the two fields. Two fields with the same intensity and relative phase $n \lambda / 2$ ( $n$ integer) lead to a linearly polarized output field with direction at \pm 45 deg with respect to the horizontal plane, as exemplified in Fig. 1(b). Similarly, if the phase is $(n+1 / 2) \lambda / 2$, the output polarization state will be fully circular with appropriate chirality, as shown in Fig. 1(a).

If diffraction effects cannot be ignored, e.g., when the distance of the two sources is larger than the Rayleigh range, the picture becomes less intuitive, as shown in Fig. 8(a)-8(c). The total degree of linear polarization $\boldsymbol{P}_{\text {lin }}$, as shown by the blue line in Fig. 8(b), changes from 0 to a maximum that depends on the $d / z_{R}$ ratio, as shown in Fig. 7, and does not reach one.

The total degree of polarization $\boldsymbol{P}$ and the intensity of the radiation [Fig. 8(a),(b) black dashed and green lines, respectively], are instead independent of the phase between the fields.

The direction of the linear polarization $\psi$ [Fig. 8(c) red line] shows a discontinuity at the phase corresponding to the minimum of linear polarization, while it is fixed to $\pm 45 \mathrm{deg}$ otherwise. The discontinuity corresponds to $\boldsymbol{P}_{\text {lin }}=0$, where the radiation has its maximum obtainable degree of circular polarization. Note that when the degree of polarization (dashed line) is $<1$, only part of the radiation is effectively converted to circularly polarized light and the rest is unpolarized radiation. The unpolarized background arises from the spatial variation of the relative
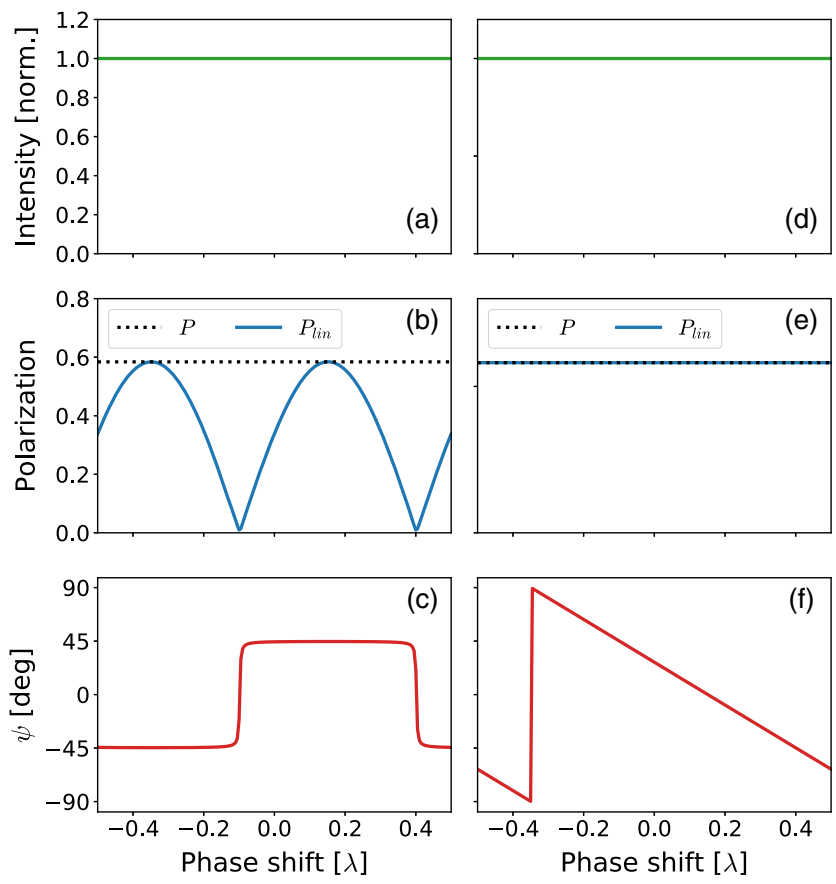

FIG. 8. Model prediction of the XPU scheme. Intensity (a, d) polarization (b, e) and direction (c, f) as a function of the phase between the two sources. The two fields have the same amplitude and linear orthogonal polarization [LV and $\mathrm{LH}$ for (a-c), CR and CL for (d-f)], while the distance between the two sources is $\sim 3 z_{R}$ and the collection aperture is $3 \sigma$ both in horizontal and vertical direction.

phase and amplitude ratio between the two interfering fields over the transverse plane.

\section{E. CL and CR fields}

The superposition of two circularly polarized fields, with opposite chirality, as shown in Fig. 1(c),(d), is another example of crossed polarization. If the effects due to the curvature of the wavefront can be neglected and the two sources have the same intensity, one would expect a fully linearly polarized resulting field with direction of polarization determined by the relative phase of the two circularly polarized fields. By changing the relative phase one could adjust the direction of the linear polarization vector at will.

If instead the plane wave approximation does not hold and diffraction plays a crucial role, [see Fig. 8(d)-(f)], the maximum degree of linear polarization will be less than one and as before will be a function of the ratio $d / z_{R}$, similar to what is shown in Fig. 7. The previous argument for the loss of the polarization also applies here and the direction of the linear polarization vector will still change linearly as the relative cross-polarization phase is modified.

\section{F. Limiting the diagnostic aperture}

The effects on polarization properties associated with different phases and field amplitudes between the crossed 
polarized fields can be further explored using the aforementioned model. We investigated the dependence upon the phase between the LV and LH fields when limiting the computation of the total polarization parameters to small regions at different locations of the wavefront, i.e., selecting a beam aperture with size $\sim \sigma / 10, \sigma$ being the beam size at the measurement location. The results are reported in Fig. 9 for $P_{\text {lin }}$ (a) and $\psi$ (b).

The curves show, for both quantities, significant shifts of the location of the maxima and minima, as well as different values of extrema, for different transverse locations along the wavefront. These results confirm that different regions of the wavefront have different polarization states. The change in the phase shift position of the extrema is a clear indication of a phase variation between the two orthogonal fields, while a change in their values is a clear indication of the variation in the amplitude ratio of the interfering fields.

Notice that in principle one could also adapt the electron beam optics along the undulator line to satisfy the condition $d / z_{R} \ll 1$, i.e., having larger transverse beam dimensions. However, a larger transverse electron beam size is less favorable for the FEL amplification process with reduced final photon flux.
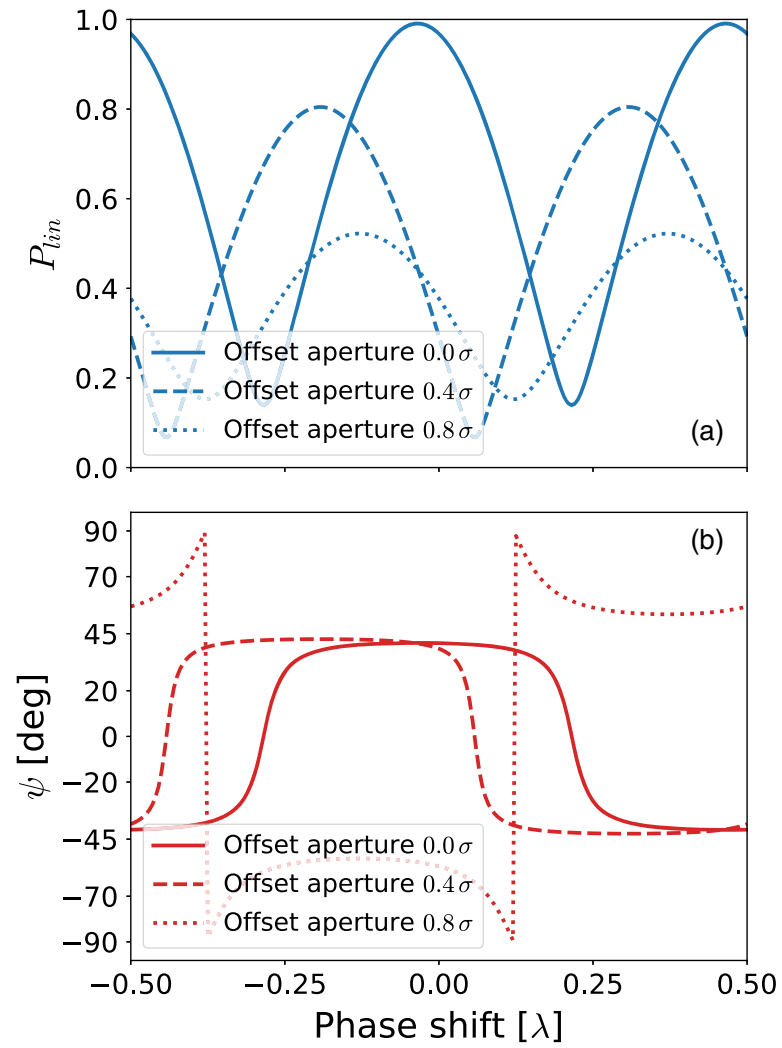

FIG. 9. Predicted degree of linear polarization (a) and direction (b) as a function of the phase between the two source fields, for different beam defining aperture position. The two fields have the same amplitude and have LV and LH polarization.

\section{EXPERIMENTAL RESULTS}

The interference effects of diffraction-limited coherent radiation beams are relevant not only for the XPU scheme but also for the simpler case of radiation emission from unipolarized undulators. The model described in Sec. II can also be used for this second case which requires the phase shifters between the subsequent undulators to be properly set in order to maximise the FEL power output. This simpler case is especially suitable to compare experimental measurements with the model's predictions since it avoids complications related to the preservation and measurement of the polarization.

\section{A. Unipolarized FEL intensity sensitivity to phase shifter setting}

The first test we performed was to change the distance between the two interfering sources. This is relatively straightforward because the undulator segments can be tuned independently in our experimental setup [see Fig. 2(a)]. Hence the distance between the sources can be incremented by simply grossly detuning one undulator module (in the experiment, the fifth module was detuned), increasing the separation distance by a factor of 2 , while maintaining the ability to scan the strength of an intervening phase shifter to change the relative phase between the two sources.

In Fig. 10 we report the (normalized) intensity of the resulting field while varying the phase between the interfering sources, for the two particular separations. The dashed lines correspond to the model's predictions. The model and the measured data agree in terms of the modulation depth for the two cases. They agree only

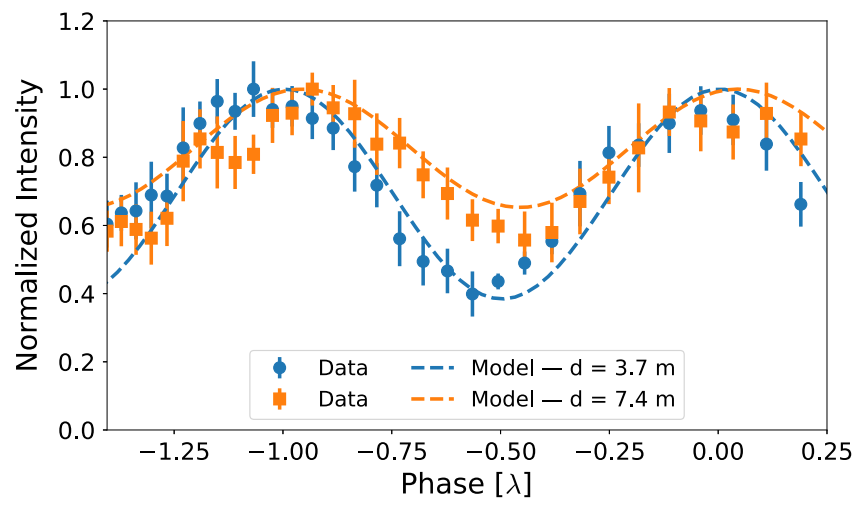

FIG. 10. Comparison between experimental data (points) and Gaussian propagation model prediction (dashed lines) for the scan of the phase between two sources in a non-XPU configuration (pure LV polarization). The distance $d$ between the two sources has been varied by opening the fifth undulator sections (see Fig. 2). The blue curve corresponds to $d=3.7 \mathrm{~m}$ while the orange one corresponds to $d=7.4 \mathrm{~m}$. Each experimental point is the average over 20 consecutive shots, the error bars are the standard deviation. 


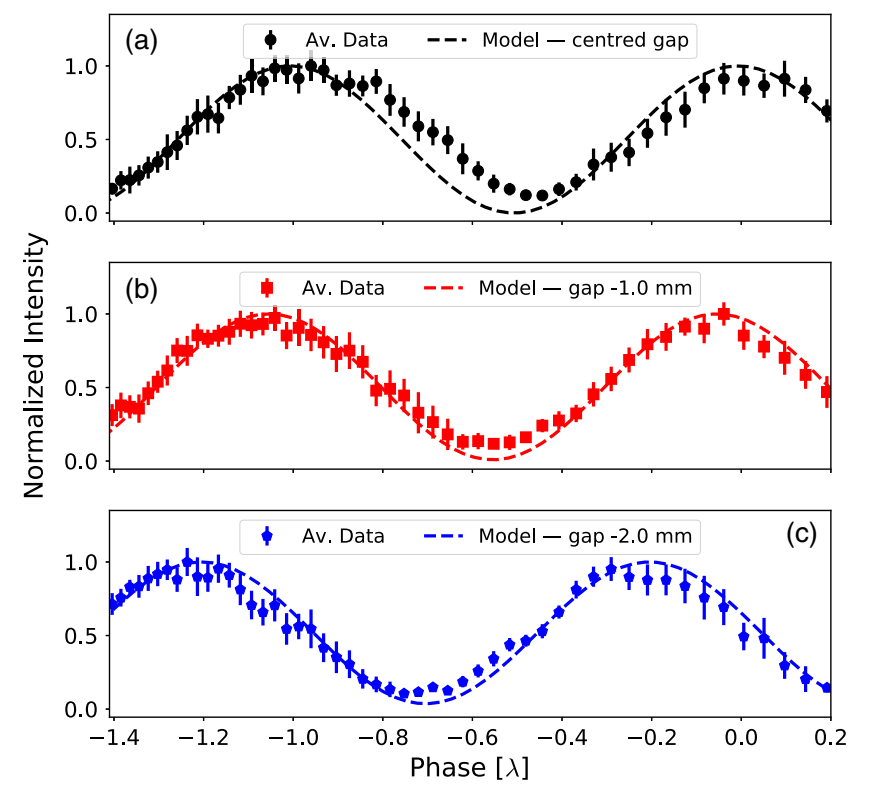

FIG. 11. Left: Experimental data (points) and model prediction (dashed lines) for the scan of the phase between two unipolarized sources, for different aperture's locations along the wavefront of the beam. All the undulators were set to linear vertical polarization. Each experimental point is the average over 20 consecutive shots, the error bars are the standard deviation.

qualitatively in terms of the location of the maxima and minima of the scan, probably due in part to the presence of gain and discrepancies with the simplistic assumption that the effective radiation sources are located at the exact center of each undulator segment.

As was true in Sec. II F for the polarization, the variation of the intensity with relative undulator phase is sensitive to the beam aperture because different regions along the wavefront will have different phase relationships between the two interfering fields.

In Fig. 11(a)-(c) we compare experimental data (dots) with the model (dashed lines), for three different aperture positions along the beam transverse front. One sees that there is a significant shift of the phases corresponding to the maximum (normalized) intensity for different aperture locations.

\section{B. LV and LH XPU fields}

In Fig. 12 we report the measured degree (blue), direction (red) of linear polarization and intensity (green) as a function of the phase between the two cross-polarized fields, for the consecutive [Fig. 12(a),(b),(c)] and distributed [Fig. 12(d),(e),(f)] undulator configuration. There were 4 undulators in LV and 2 in LH polarization for the consecutive scheme, while all 6 segments were set in alternating polarizations for the distributed scheme [see Fig. 2(b),(c)]. The mean and standard deviations were obtained from 40 shots, acquired at each value of the phase between the two fields. The diagnostic apertures were fully open during the acquisition. To determine the amplitudes of
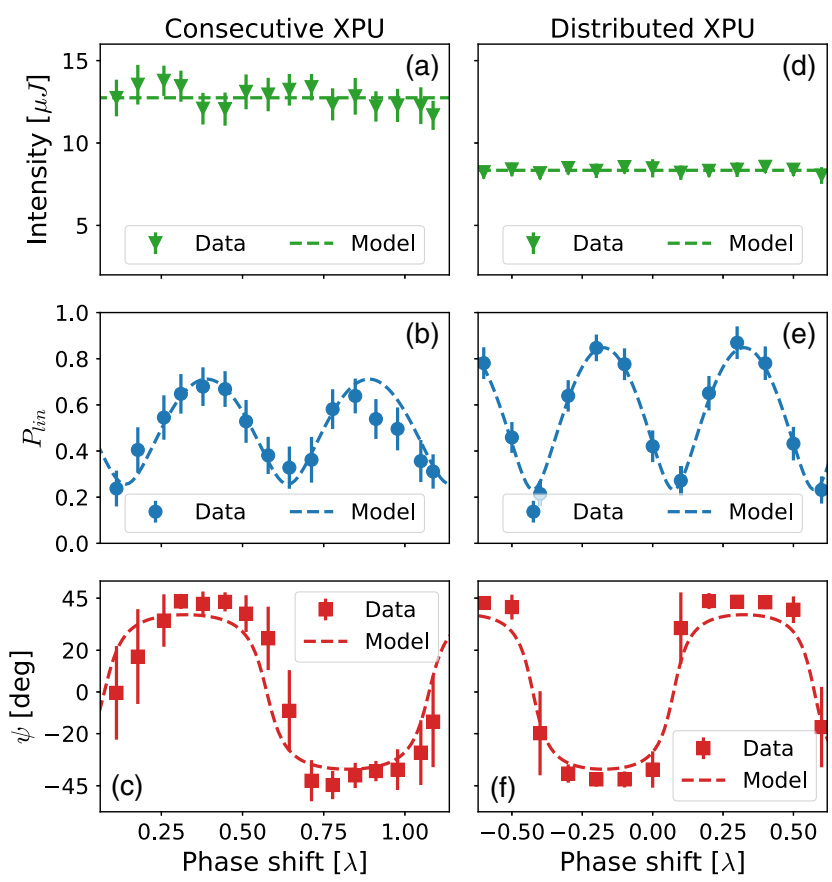

FIG. 12. Scan of the phase between LV and LH crossed polarized fields. The degree (b, e, blue) and direction (c, f, red) of the linear polarization vector are reported as a function of the phase between the two fields, together with the intensity of the FEL radiation (a, d, green). Experimental data (markers) and Gaussian propagation model predictions (dashed lines) are reported for the consecutive $(a, b, c)$ and the distributed $(d, e, f)$ schemes. The model was obtained using a set of parameters obtained by the gain curve measurements for the intensity of the fields and the electron beam size measurements for the waist sizes of the sources. The consecutive scheme was obtained using 4 undulators in LV polarization and 2 in $\mathrm{LH}$, while the distributed scheme was implemented using alternating polarization undulators.

the fields used in the model, we measured the FEL intensity as a function of the number of undulators (i.e., a gain curve), obtained in the different configurations (data not shown). The Rayleigh length $z_{R}$ has been derived from electron beam transverse size measurements for the waist sizes of the sources. It is fixed to $1.32 \mathrm{~m}$ in all the reported results in the following.

By changing the phase in this linear crossed polarized undulator scheme one can obtain light with linear polarization at 45 deg angle at the maxima of $\boldsymbol{P}_{\text {lin }}$ or circularly polarized light at the minima of $\boldsymbol{P}_{\text {lin }}$. According to the predictions of the Gaussian propagation model, we expect that the total output radiation will not reach a degree of polarization $\boldsymbol{P}=1$ due to off-axis curvature of the wavefronts and partial overlap of the two cross-polarized fields.

One can see in Fig. 12(b),(e) that for both schemes $\boldsymbol{P}_{\text {lin }}$ is oscillating as a function of the phase, with maximum values of approximately 0.8 and 0.7 for the distributed and consecutive schemes, respectively. While for the distributed configuration the predictions of the theoretical model agree 
well in value and shape, for the consecutive configuration the maxima are different. We believe the difference can be explained by imperfect circular polarization (i.e., an elliptical polarization residual) in the two sources. Note that this behavior agrees with the previous FERMI results found for the "pure" polarization case [18]. The presence of a residual ellipticity is further confirmed by looking at the intensity of the FEL as a function of the phase, which shows oscillations in the consecutive case. The direction of linear polarization $\psi$ is oscillating from $\sim-40 \mathrm{deg}$ to $\sim 40 \mathrm{deg}$ for both configurations, see Fig. 12(c),(f).

The experimental results of the distributed XPU configuration confirm its clear advantage over the consecutive configuration. We believe this is due in part to a lower sensitivity to monotonic variations in $z$ of electron beam parameters such as the bunching, size, transverse offset, and tilt.

In Fig. 13 we show the phase shifter scan results when the measurement aperture is closed to $1 \times 1 \mathrm{~mm}^{2}$ and sampled at different transverse locations. Both the degree $(a, c)$ and direction $(b, d)$ of the linear polarization vector show significant differences between the consecutive [Fig. 13(a),(b)] and distributed [Fig. 13(c),(d)] schemes. The curves correspond to full aperture (black) and two different positions of a $1 \times 1 \mathrm{~mm}$ beam-defining aperture. The consecutive scheme shows a significant variation of the trends for both $P_{l i n}$ and $\psi$ at different aperture locations,
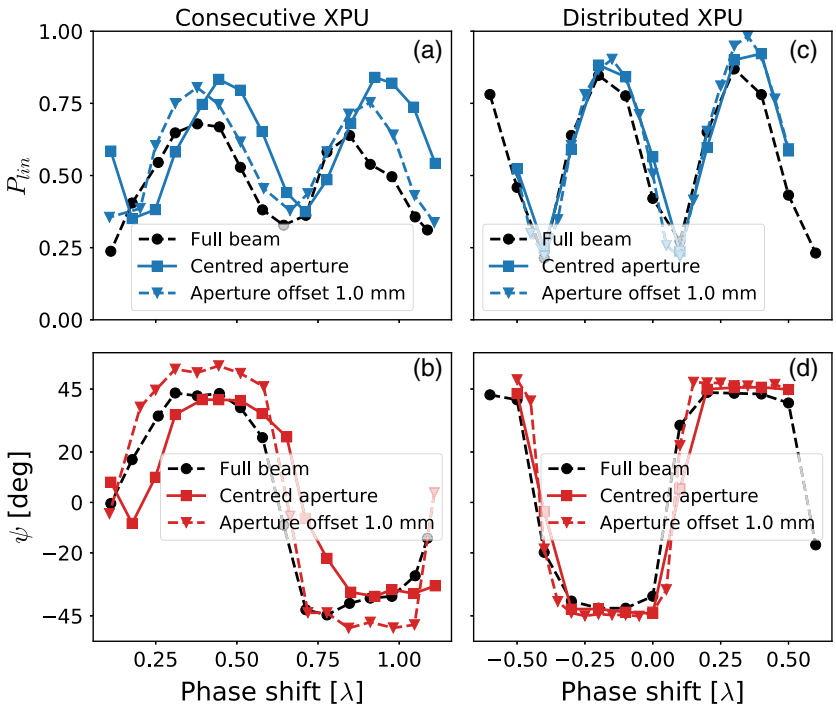

FIG. 13. Scan of the phase between the horizontal and vertical polarized fields for the consecutive $(a, b)$ and distributed (c, d) XPU configurations. The degree (blue) and direction (red) of the linear polarization vector are reported as a function of the phase between the two fields. The curves correspond to full aperture (black) and two different positions of a $1 \times 1 \mathrm{~mm}$ beam defining aperture. The data were obtained as the average of 40 consecutive shots at the same phase shift; the uncertainties are similar to Fig. 12 and are not shown for better clarity. For a comparison with the model predictions, see Fig. 9. including different values and positions of the extrema. In particular, the maximum polarization value is much greater for the limited apertures. The distributed scheme results are instead characterized by a far more uniform behavior as a function of the aperture position. The phases corresponding to the extreme values are similar to those found with the fully open aperture.

The change in the position of the extrema is a clear indication of the phase variation between the two orthogonal fields along the beam wavefront, while the change in their value is an indication of the variation of their amplitude ratio. In both cases the measured results are in satisfactory agreement with the model, as displayed in Fig. 9.

\section{CR and CL fields}

The XPU scheme can be also implemented by superposing two circularly polarized fields with opposite chirality to obtain linearly polarized light at an arbitrary angle. As discussed in the next subsection, this approach can be used to suppress harmonic emission on axis.

Figure 14 displays experimental results for such a configuration. The degree (blue) and the direction (red) of the
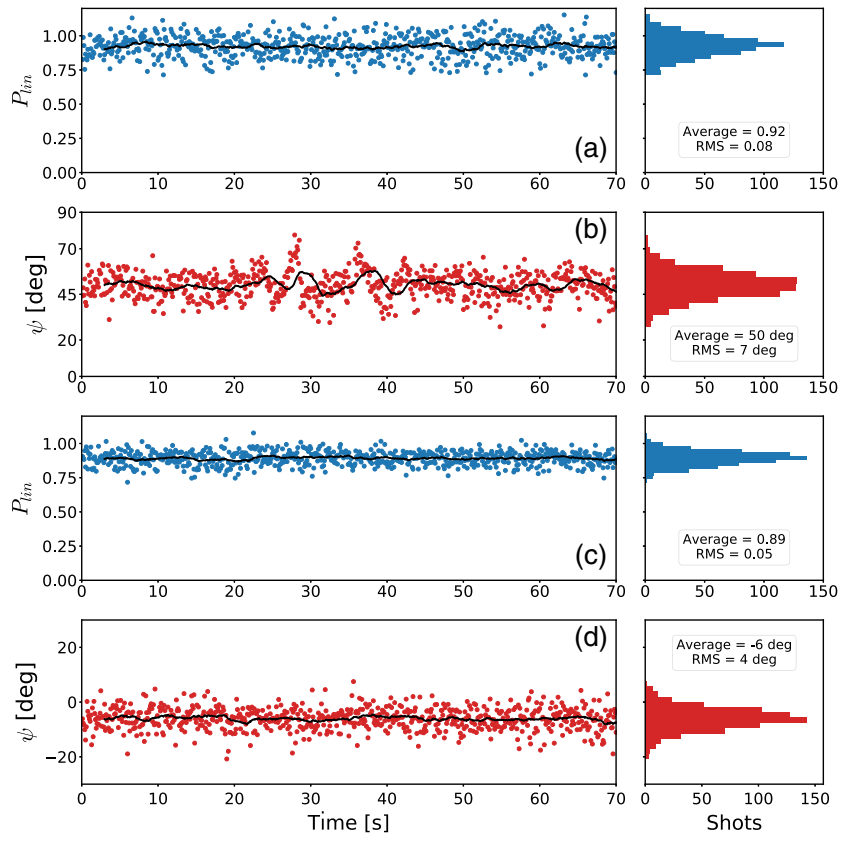

FIG. 14. Degree and direction of the linear polarization component for the circular XPU. Panels $(\mathrm{a}, \mathrm{b})$ refer to the consecutive scheme [Fig. 2(b)], while (c, d) refer to the distributed scheme [Fig. 2(c)]. The lines represent the moving average over 30 shots. The data were acquired selecting a $1 \times 1 \mathrm{~mm}^{2}$ aperture, centered on the beam axis. The difference in the absolute value of $\psi$ between the two configurations is only due to the particular settings of the phase shifters in the two measurements, and can be freely varied as reported in Fig. 16. The data for the full aperture case is reported in [18]. 
linear polarization vector are plotted for a sequence of 700 consecutive shots. Data with a degree of linear polarization that exceeds one is physically not possible but algebraically correct for the used model as imprinted in the statistical fluctuations on the e-tof signal [18]. The data in Fig. 14 was obtained by reducing the diagnostic aperture to a $1 \times 1 \mathrm{~mm}^{2}$ size, centered on the beam axis. Fig. 14(a),(b) refer to the consecutive configuration, while Fig. 14(c),(d) refer to the distributed one. The consecutive scheme was implemented using 4 undulators with one helicity and 1 with the opposite helicity, while the distributed scheme used all 6 available devices, with alternating helicities. The data can be compared to [18] where we measured the properties of the full beam, without limiting apertures. As predicted by the Gaussian propagation model, limiting the collection aperture of the radiation can be used to select a region of the wavefront with nearly uniform polarization properties. The difference in the absolute value of $\psi$ between the two configurations is only due to the particular settings of the phase shifters in the two measurements and can be freely adjusted, e.g., as shown in Fig. 16 for the distributed XPU scheme.

The average degree of linear polarization is $\sim 0.9$ for both cases. As previously remarked in [18], a significant difference between the two schemes is evident when examining the stability of direction of the polarization vector. The RMS fluctuation levels are $\sim 4 \mathrm{deg}$ for the distributed scheme but $\sim 7 \mathrm{deg}$ for the consecutive scheme. This difference is important, as the fluctuations in the distributed scheme have similar amplitude as the "pure" polarization state and are within the estimated instrumental error. The significantly larger fluctuation level of the consecutive configuration strongly suggests that the direction of the polarization is changing on a shot-to-shot basis as a consequence of e-beam variations. The $\mathrm{CL}+\mathrm{CR}$ results confirm what was found previously in the linear case showing the advantage of the distributed configuration.

We also measured the dependence of the polarization properties transversely along the wavefront of the beam, again by changing the beam defining aperture center and dimensions. The results are reported in Fig. 15 for the intensity (green), $P_{\text {lin }}$ (blue) and $\psi($ red). Figure 15(a),(b),(c) refer to the consecutive scheme, while Fig. 15(d),(e),(f) refer to the distributed scheme. The value for each quantity (shown inside the squares) was obtained as an average over 500 shots. In both configurations one can clearly observe a variation of the degree and direction of the linear
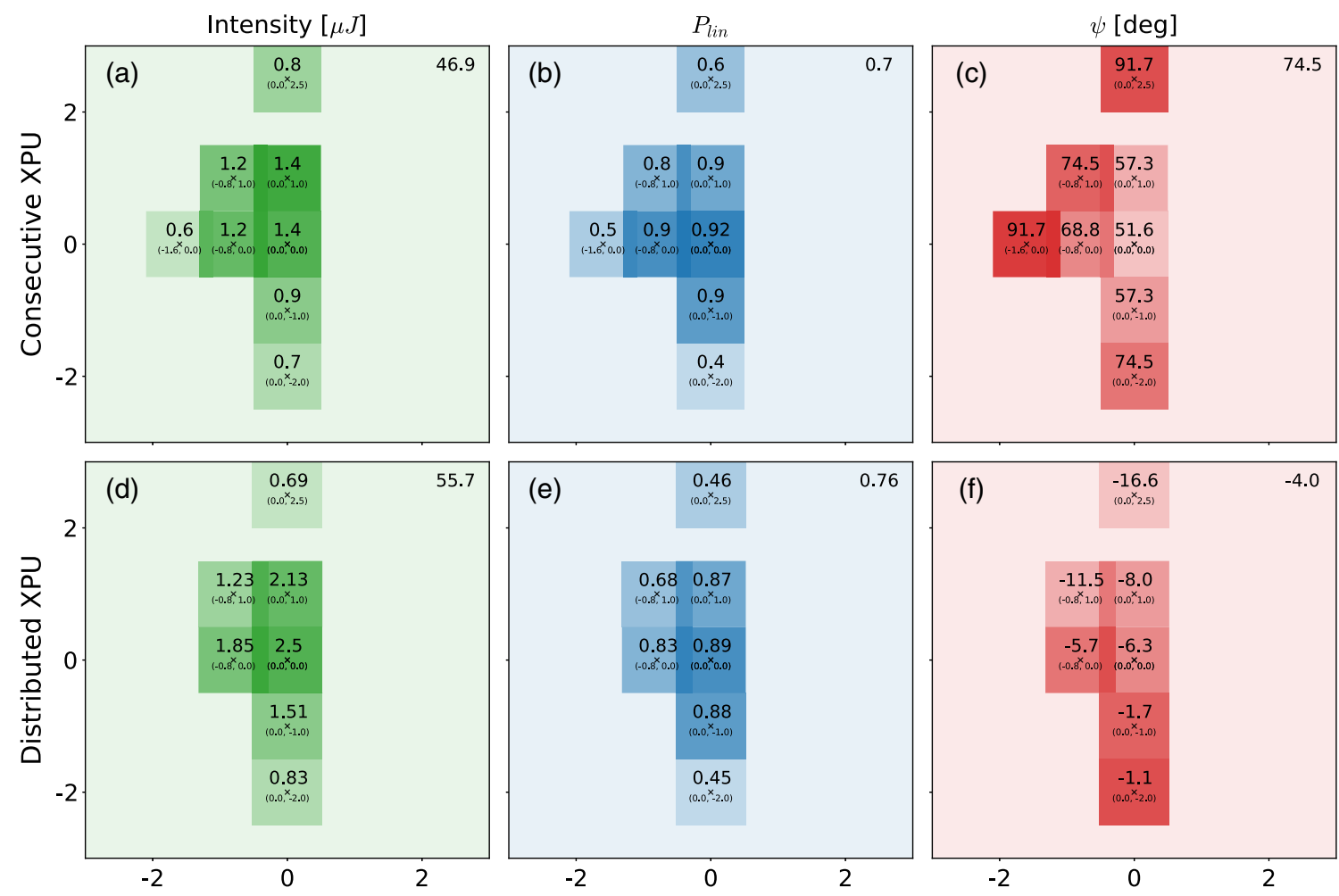

FIG. 15. Intensity (in $\mu \mathrm{J}$ ), degree and direction (in deg) of the linear polarization for the circular XPU for different beam defining aperture positions (in $\mathrm{mm})$. (a, b, c) are relative to the consecutive scheme, while (d, e, f) refer to the distributed scheme. The value of each quantity is reported in the boxes that represent each different aperture position (on the top right corner for the larger aperture). One can clearly see that in both configurations the polarization properties change along the wavefront, due to phase and amplitude variations between the interfering fields. The reported values represent the average over 500 shots. The different rate of change for $\psi$ between the horizontal and vertical direction suggests either that the photon beam was slightly elliptical during our measurements, or that the different sources were not perfectly aligned. 
polarization vector at different locations of the beam wavefront. The maximum $P_{\text {lin }}$ occurs at the on-axis observation point. The degree of polarization decays when moving offaxis, similar to the model prediction, see Fig. 6. Also the direction of the polarization vector changes along the wavefront. This confirms that the decrease in the overall polarization observed for the XPU scheme is due to variations of the polarization properties along the wavefront of the beam as a consequence of the relative phases and amplitudes of the two interfering waves. This variation is important when the distance between the orthogonal sources is larger than the Rayleigh range of the radiation, as in the present case.

Finally, in Fig. 16 we report the results of an experiment confirming the possibility of controlling the direction of the linear polarization by varying the phase between the two consecutive sets of undulators. The direction of linear polarization is reported, as a function of the phase between the two circularly polarized fields, in distributed configuration. As one can see, the direction of the linear polarization vector can be arbitrarily adjusted by changing the phase between the two fields and has a linear dependence with the phase shifter itself. If one then swaps the chirality of the two undulator groups, the same linear dependence can be obtained with opposite sign. The data were collected with open diagnostic aperture. In both cases the degree of linear polarization, $\sim 0.8$, was independent of the phase, within the measurement uncertainty.

This result confirms the ability to finely control the polarization state using the XPU scheme. The possibility to arbitrarily modify the direction of linear polarization solely

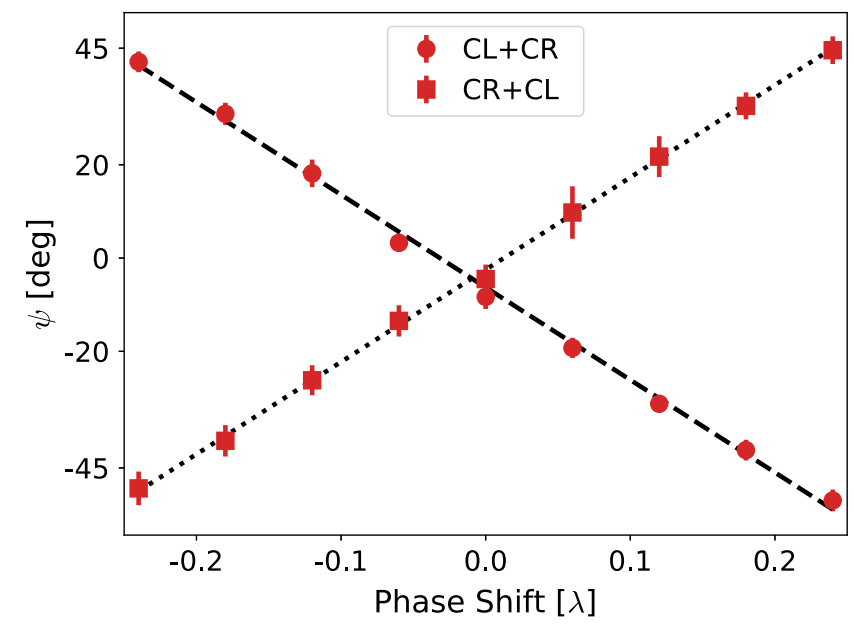

FIG. 16. Direction of the linear polarization vector as a function of the phase between the circularly polarized fields with opposite chirality. The distributed scheme was used. The dotted and dashed lines show the expected linear dependence. By swapping the chiralities of each undulator the same linear dependence can be observed, but with opposite sign. The data were obtained by the statistical analysis (average and standard deviation) of 40 consecutive shots at the same phase shift value. through a phase shifter change represents a powerful extension of the FEL capabilities for users interested in rapid experimental variation of the polarization vector direction, as the APPLE-II undulators installed at FERMI by themselves can produce linearly polarized light only along the horizontal or vertical planes.

\section{Harmonic suppression}

Another attractive possibility enabled by the XPU scheme is the ability to produce linearly polarized radiation without on-axis harmonic emission by superimposing CR and CL polarized sources. Normally, when one is interested in linearly polarized light, an undulator in simple, unidirectional linear polarization is used. The output radiation is then contaminated by emission of on-axis, odd harmonics of the fundamental undulator radiation. Typical intensities associated with the third harmonic are a factor $\sim 10^{-3}$ of the fundamental, but they could significantly limit certain experiments. For example, if one is interested in nonlinear processes induced by the absorption of three photons at the fundamental wavelength [19]: the linear signal induced by the absorption of the harmonic emission can be orders of magnitude larger than the nonlinear one. Using circularly unipolarized undulators can limit the harmonic content, as the harmonic radiation is emitted off-axis [35,36]. However in this case the fundamental is in a circular polarization state. The XPU CL + CR scheme can be a viable option for linearly polarized light without the unwanted on-axis harmonic emission.

In order to confirm this prediction we looked at the third harmonic radiation strength using the FERMI's downstream radiation spectrometer, while the undulators where tuned for the XPU scheme. The images shown in Fig. 17(a)-(b) compare the emission at $7.95 \mathrm{~nm}$, the third harmonic of the wavelength used during the experiments, when all the undulators are tuned to emit linearly polarized radiation [orange curve and false color image (a)] and in the XPU CR and CL scheme [blue curve and image (b)].

While the emission at the fundamental wavelength was similar in the two cases, with $\sim 20 \mu \mathrm{J}$ pulse energy, the on-axis 3rd harmonic radiation is strongly suppressed in the case of the XPU scheme. The XPU scheme was realized in the distributed configuration, but similar results were also confirmed with the consecutive configuration. Off-axis harmonics can be preferentially spatially filtered out using the beam defining aperture that, as we have seen above, also increases the degree of linear polarization of the transmitted radiation.

\section{E. XPU scheme and SASE}

Finally we tested both the consecutive and the distributed configuration with SASE radiation [37]. In the case of FERMI, the total undulator length is too short to achieve saturation; hence the measurements reported in the following refer to a condition still deep in the exponential gain 


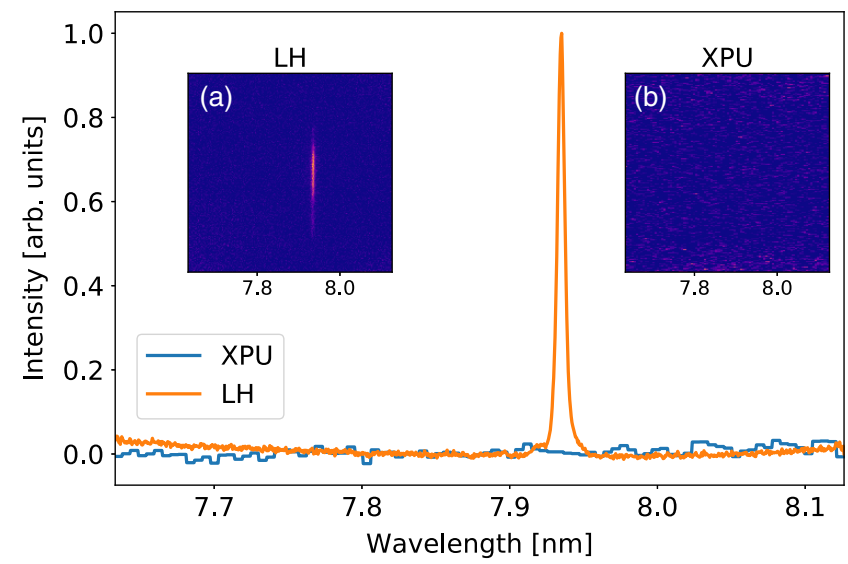

FIG. 17. Suppression of the harmonic signal in the XPU scheme. [orange curve and false color image in (a)] Third harmonic spectrum of the emission when the undulator is tuned in LH polarization and for the XPU alternated configuration, when two circularly polarized fields are superimposed in order to produce linearly polarized radiation [blue curve and image in (b)]. A harmonic signal is clearly present for the pure polarization state and not detectable in the XPU scheme. The intensities of the first harmonic were comparable in the two cases $(\sim 20 \mu \mathrm{J}$ pulse energy). The spectra were accumulated for 11 shots in both cases.

regime, employing the $\mathrm{CR}$ and $\mathrm{CL}$ polarization XPU scheme to produce linearly polarized radiation. The beam parameters were similar to those reported in [37], except for the final wavelength of $23.8 \mathrm{~nm}$ (gain length $1.6 \mathrm{~m}$ for the consecutive and $1.5 \mathrm{~m}$ for the distributed configuration), the same as for the seeding results reported earlier. In Fig. 18 the consecutive (top two panels) and the distributed configuration (bottom two panels) results are shown for the degree (blue) and direction of linear polarization (red).

For SASE the obtained degree of linear polarization when superposing two circularly polarized fields is lower than the one obtained with the seeding for the same collection aperture. One can also notice that the consecutive configuration leads to an average $\boldsymbol{P}_{\text {lin }}$ of 0.5 , while the distributed configuration is characterized by an average $\boldsymbol{P}_{\text {lin }}$ of 0.27 . This result is opposite to the one observed for the seeded case, where the distributed scheme can lead to a higher degree of linear polarization. Furthermore we did not observe a change in the polarization properties by limiting the collection aperture of the FEL light. In our understanding, these results indicate that the limiting factor for the XPU in SASE is the longitudinal coherence of the radiation, rather than the transverse change of phase and amplitude of the two superimposing fields along the wavefront. Similar results have been reported for the LCLS facility in SASE conditions [38].

\section{SUMMARY}

We developed a simple but flexible model, based on Gaussian mode radiation beams, that can be used to study the interference of the radiation fields emitted by different monochromatic sources, e.g., in the XPU scheme. We showed that the maximum obtainable degree of polarization critically depends on the ratio of the source separation distance to the effective Rayleigh range.

We also investigated the dependence of the scheme on the phase between the two sources with both linear and circular orthogonal polarization. The impact of polluting effects on the scheme has been studied. We showed that the predicted polarization properties change along the beam wavefront due to changes in the phasing and/or in the amplitude ratio of the interfering fields, leading, on average, to a loss of polarization.

The experimental results here reported help in improving the understanding of the XPU scheme and its applicability at FERMI in the XUV wavelength range, in both the consecutive and distributed configuration geometry, superimposing either two linearly cross-polarized fields or two circularly polarized ones.

The stability of both schemes has been measured again with respect to the direction of the polarization vector and the degree of linear polarization. Also when considering only a small portion of the beam, we observed that the distributed scheme is characterized by a higher shot-toshot stability, mainly in terms of the polarization vector
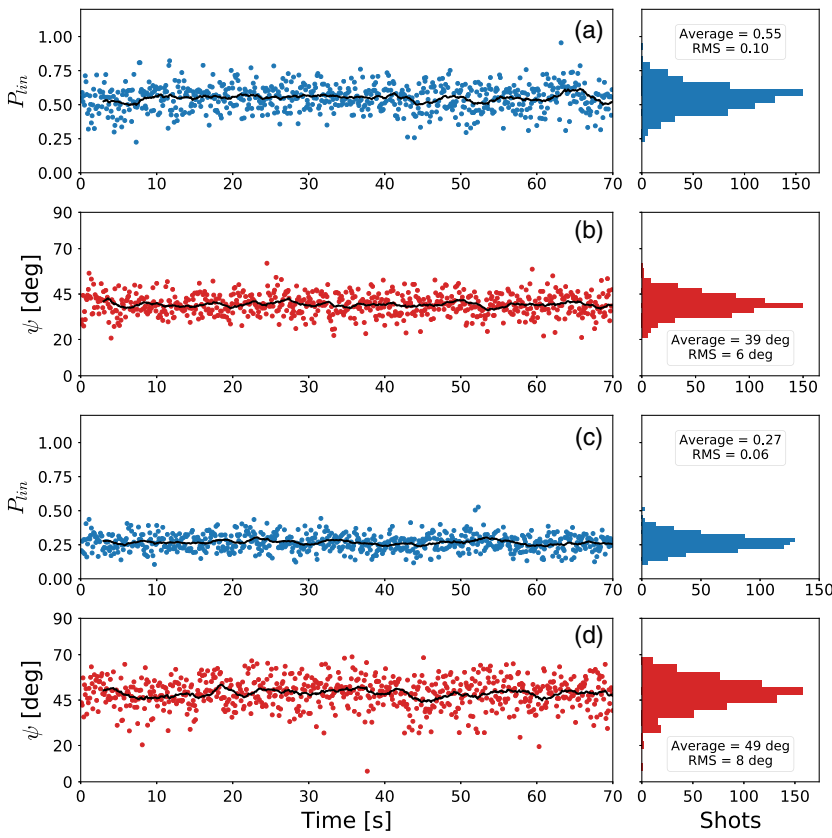

FIG. 18. Degree and direction of the linear polarization component for the XPU with circular polarization in SASE. $(\mathrm{a}, \mathrm{b})$ refer to the consecutive configuration, obtained using 4 undulators with $\mathrm{CR}$ and 1 undulator with CL polarization. (c, d) refer to the distributed configuration with 6 undulators. The lines represent the moving average over 30 shots of the reported quantities. Note that, in the case of SASE, the distributed configuration leads to a lower degree of linear polarization than the consecutive one. 
direction $\boldsymbol{\psi}$. We confirmed that the degree of linear polarization displays the same fluctuation level that was obtained with the pure LH undulator, below the instrumental noise [18]. An increase of a factor about two in the amplitude of the fluctuations of $\boldsymbol{\psi}$ has been observed for the consecutive XPU scheme compared to the distributed case, as reported in [18] for the full beam case. Both circularly polarized XPU configurations are able to produce a high degree of horizontally polarized light.

The good agreement with theoretical predictions of this simple model indicates a high degree of both longitudinal and transverse coherence of the FEL pulses which generate the polarized light. In fact, if SASE radiation is considered, the performance of the XPU scheme is poor and, in our understanding, limited by the reduced longitudinal coherence of the SASE pulses relative to the externally seeded case.

We also demonstrated the capability of the XPU scheme to produce linearly polarized radiation free of on-axis harmonic components, providing a remarkable benefit to nonlinear experiments where harmonic contamination is detrimental.

The measured levels of polarization are sufficient for a wide range of user experiments requiring polarization control. Limiting the beam collection aperture leads to an even larger degree of polarization with values of 0.9 obtainable. The direction of the linear polarization vector can also be varied at will.

\section{ACKNOWLEDGMENTS}

We thank Andreas Beckmann for the software support related to the data acquisition of the polarimeter, Markus Ilchen for the assistance in setting up the polarimeter instrumentation, and William M. Fawley and Zhirong Huang for helpful comments.

[1] D. Desiderio et al., The ELETTRA circular polarization beamline and electromagnetic elliptical wiggler insertion device, Synchrotron Radiat. News 12, 34 (1999).

[2] J. Bahrdt, W. Frentrup, A. Gaupp, M. Scheer, W. Gudat, G. Ingold, and S. Sasaki, Elliptically polarizing insertion devices at BESSY II, Nucl. Instrum. Methods Phys. Res., Sect. A 467-468, 21 (2001).

[3] C. Benabderrahmane et al., First SOLEIL insertion devices are ready to produce photons for users, Nucl. Instrum. Methods Phys. Res., Sect. A 575, 33 (2007).

[4] L. Nahon, N. de Oliveira, G. A. Garcia, J.-F. Gil, B. Pilette, O. Marcouillé, B. Lagarde, and F. Polack, DESIRS: A state-of-the-art VUV beamline featuring high resolution and variable polarization for spectroscopy and dichroism at SOLEIL, J. Synchrotron Radiat. 19, 508 (2012).

[5] H. Wang, P. Bencok, P. Steadman, E. Longhi, J. Zhu, and Z. Wang, Complete polarization analysis of an APPLE-II undulator using a soft X-ray polarimeter, J. Synchrotron Radiat. 19, 944 (2012).
[6] J. Viefhaus, F. Scholz, S. Deinert, L. Glaser, M. Ilchen, J. Seltmann, P. Walter, and F. Siewert, The variable polarization XUV beamline P04 at PETRA III: Optics, mechanics and their performance, Nucl. Instrum. Methods Phys. Res., Sect. A 710, 151 (2013).

[7] K.-J. Kim, Polarization characteristics of synchrotron radiation sources and a new two undulator system, Nucl. Instrum. Methods Phys. Res., Sect. A 222, 11 (1984).

[8] K.-J. Kim, Circular polarization with crossed-planar undulators in high-gain FELs, Nucl. Instrum. Methods Phys. Res., Sect. A 445, 329 (2000).

[9] Y. Li et al., Study of controllable polarization sase fel by a crossed-planar undulator, in Proceedings of FELO8, Gyeongju, Korea, TUPPH004 (2008), http://accelconf .web.cern.ch/accelconf/fel2008/papers/tupph004.pdf.

[10] E. A. Schneidmiller and M. V. Yurkov, Obtaining high degree of circular polarization at X-ray free electron lasers via a reverse undulator taper, Phys. Rev. Accel. Beams 16, 110702 (2013).

[11] J. Bahrdt, A. Gaupp, W. Gudat, M. Mast, K. Molter, W. B. Peatman, M. Scheer, Th. Schroeter, and Ch. Wang, Circularly polarized synchrotron radiation from the crossed undulator at BESSY, Rev. Sci. Instrum. 63, 339 (1992).

[12] F. U. Hillebrecht, H. B. Rose, T. Kinoshita, Y. U. Idzerda, G. van der Laan, R. Denecke, and L. Ley, Photoelectron Diffraction in Magnetic Linear Dichroism, Phys. Rev. Lett. 75, 2883 (1995).

[13] E. Allaria, B. Diviacco, C. Callegari, P. Finetti, B. Mahieu, J. Viefhaus, M. Zangrando, G. De Ninno, G. Lambert et al., Control of the Polarization of a Vacuum-Ultraviolet, HighGain, Free-Electron Laser, Phys. Rev. X 4, 041040 (2014).

[14] E. Roussel et al., Polarization characterization of soft x-ray radiation at FERMI FEL-2, Photonics 4, 29 (2017).

[15] https://emanim.szialab.org/index.html.

[16] Y. K. Wu, N. A. Vinokurov, S. Mikhailov, J. Li, and V. Popov, High-Gain Lasing and Polarization Switch with a Distributed Optical-Klystron Free-Electron Laser, Phys. Rev. Lett. 96, 224801 (2006).

[17] H. Deng, T. Zhang, L. Feng, C. Feng, B. Liu, X. Wang, T. Lan, G. Wang, W. Zhang et al., Polarization switching demonstration using crossed-planar undulators in a seeded freeelectron laser, Phys. Rev. ST Accel. Beams 17, 020704 (2014).

[18] E. Ferrari et al., Single shot polarization characterization of XUV FEL pulses from crossed polarized undulators, Sci. Rep. 5, 13531 (2015).

[19] K. C. Prince et al., Coherent control with a short-wavelength free-electron laser, Nat. Photonics 10, 176 (2016).

[20] G. De Ninno et al., Single-shot spectro-temporal characterization of XUV pulses from a seeded free-electron laser, Nat. Commun. 6, 8075 (2015).

[21] D. Gauthier et al., Spectrotemporal Shaping of Seeded FreeElectron Laser Pulses, Phys. Rev. Lett. 115, 114801 (2015).

[22] E. Allaria et al., Highly coherent and stable pulses from the FERMI seeded free-electron laser in the extreme ultraviolet, Nat. Photonics 6, 699 (2012).

[23] E. Allaria et al., The FERMI free-electron lasers, J. Synchrotron Radiat. 22, 485 (2015).

[24] J. Viefhaus, An online diagnostic unit for FEL photon parameter determination and optimization, in FEL 2015: Proceedings of the 37th International Free-Electron Laser 
Conference, Dejeon, South Korea, WED02 (2015), http:// accelconf.web.cern.ch/AccelConf/FEL2015/talks/wed02_ talk.pdf.

[25] C. Svetina et al., The low density matter (LDM) beamline at FERMI: Optical layout and first commissioning, J. Synchrotron Radiat. 22, 538 (2015).

[26] Y. Ding and Z. Huang, Statistical analysis of crossed undulator for polarization control in a self-amplified spontaneous emission free electron laser, Phys. Rev. Accel. Beams 11, 030702 (2008).

[27] E. Ferrari et al., Widely tunable two-colour seeded freeelectron laser source for resonant-pump resonant-probe magnetic scattering, Nat. Commun. 7, 10343 (2016).

[28] S. Sasaki, Analyses for a planar variably-polarizing undulator, Nucl. Instrum. Methods Phys. Res., Sect. A 347, 83 (1994).

[29] G. Geloni, V. Kocharyan, and E. Saldin, Improvement of the crossed undulator design for effective circular polarization control in X-ray FELs, arXiv:1101.4085.

[30] T. Tanaka and H. Kitamura, Improvement of crossed undulator for higher degree of polarization, AIP Conf. Proc. 705, 231 (2004).

[31] S. Yamamoto, Y. Senba, T. Tanaka et al., New soft X-ray beamline BL07LSU at SPring-8, J. Synchrotron Radiat. 21, 352 (2014).
[32] E. Saldin and E. Schneidmiller, and M. V. Yurkov, The Physics of Free Electron Lasers (Springer Science, New York, 2000)

[33] O. Svelto, Principles of Lasers, 5th ed. (Springer, New York, 2010).

[34] H. G. Berry, G. Gabrielse, and A. E. Livingston, Measurement of the Stokes parameters of light, Appl. Opt. 16, 3200 (1977).

[35] M. J. Schmitt and C. J. Elliott, Even-harmonic generation in free-electron lasers, Phys. Rev. A 34, 4843 (1986).

[36] C. Spezzani, E. Allaria, M. Coreno, B. Diviacco, E. Ferrari, G. Geloni, E. Karantzoulis, B. Mahieu, M. Vento, and G. De Ninno, Coherent Light with Tunable Polarization from Single-Pass Free-Electron Lasers, Phys. Rev. Lett. 107, 084801 (2011).

[37] G. Penco, E. Allaria, G. De Ninno, E. Ferrari, and L. Giannessi, Experimental Demonstration of Enhanced SelfAmplified Spontaneous Emission by an Optical Klystron, Phys. Rev. Lett. 114, 013901 (2015).

[38] H.-D. Nuhn et al., Commissioning of the Delta polarizing undulator at LCLS, in Proceedings of FEL2015, Daejeon, Korea, WED01 (2015), http://accelconf.web.cern.ch/ AccelConf/FEL2015/papers/wed01.pdf. 